\title{
BMJ Open Prevalence and risk factors of anxiety and depression among patients with breast cancer: a protocol for systematic review and meta-analysis
}

\author{
Jie Li (D) , ${ }^{1}$ Feng Zhang (D) , ${ }^{1}$ Wenchun Wang, ${ }^{2}$ Rizhao Pang, ${ }^{2}$ Jiancheng Liu, ${ }^{2}$ \\ Qiuhong Man, ${ }^{3}$ Anren Zhang ${ }^{4}$
}

To cite: Li J, Zhang F, Wang W, et al. Prevalence and risk factors of anxiety and depression among patients with breast cancer: a protocol for systematic review and meta-analysis. BMJ Open 2021;11:e041588. doi:10.1136/ bmjopen-2020-041588

- Prepublication history for this paper is available online. To view these files, please visit the journal online (http://dx.doi. org/10.1136/bmjopen-2020041588).

$\mathrm{JL}$ and FZ contributed equally.

Received 13 June 2020 Revised 15 December 2020 Accepted 14 January 2021

\section{Check for updates}

(c) Author(s) (or their employer(s)) 2021. Re-use permitted under CC BY-NC. No commercial re-use. See rights and permissions. Published by BMJ.

${ }^{1}$ Acupuncture and Tuina School, Chengdu University of Traditional Chinese Medicine, Chengdu,

China

${ }^{2}$ The General Hospital of Western Theater Command, Chengdu, China

${ }^{3}$ Shanghai Fourth People's Hospital Affiliated to Tongji University School of Medicine, Shanghai, China

${ }^{4}$ Department of Rehabilitation Medicine, Shanghai Fourth People's Hospital Affiliated to Tongji University School of Medicine, Shanghai, China

Correspondence to

Dr Anren Zhang;

anren0124@163.com

\section{ABSTRACT}

Background Patients with breast cancer often experience severe psychological distress, especially anxiety and depression, leading to poorer quality of life, shortened survival time and increased mortality.

The objective of the review will be to summarise data on the prevalence and risk factors of anxiety and depression in patients with breast cancer.

Methods and analysis Two reviewers will be applied in seven databases, including Web of Science, PubMed, EMBASE, Wan Fang Data Knowledge Service Platform, Chinese Biomedical Literature Database, Chinese Scientific Journal Database (VIP database), China National Knowledge Infrastructure and for studies on the prevalence and risk factors of depression in patients with breast cancer, which should be published from inception to Feb 2020 in English, Chinese, French and Spanish. The selection of studies, data extraction and risk of bias assessment will be done independently by two reviewers. Data synthesis will be carried out using RevMan V.5.3 software. The heterogeneity will be determined by the $I^{2}$ test. Publication bias will be evaluated by generating a funnel plot and performing the Begg and Egger test. The quality of the systematic review will be assessed using the Grading of Recommendations Assessment, Development and Evaluation Tool criteria.

Ethics and dissemination No ethical approval is required. This protocol will not involve individual patient information and endangering participant rights. The results will be reported in a peer-reviewed journal or disseminated in relevant conferences.

OSF registration number DOI 10.17605/OSF.IO/D6A4P.

\section{INTRODUCTION}

Breast cancer in women is increasing worldwide. The Cancer Statistics ${ }^{1}$ produced the three most common cancers, including breast cancer, lung cancer and colorectal cancer, accounting for $50 \%$ of all new diagnoses. Prevalence of breast cancer alone accounts for $30 \%$ of female cancers and has been listed as the most frequent cancer diagnosis of female malignancy. It is known as global
Strengths and limitations of this study

- The study aims to summarise data on the prevalence and risk factors of anxiety and depression in patients with breast cancer.

- The selection of studies, data extraction and risk of bias assessment will be done independently by two reviewers.

- The quality of the systematic review will be assessed using the Grading of Recommendations Assessment, Development and Evaluation criteria.

- In this review, only studies published in English or Chinese will be considered, which may cause a potential risk of publication bias.

- Different measurements and tools might lead to inconsistent levels of outcomes.

cancer statistics $^{2} ; 2088849$ new cases were reported in 2018.

Published epidemiological reports around the world ${ }^{3}$ show that there is a significant increase in the death rate from breast cancer over the past two decades. Approximately 0.5 million people worldwide die from metastatic breast cancer every year even after receiving many therapies. Patients with breast cancer suffer from psychological and physical cancerrelated stressors, which may affect patients for many years after treatment. ${ }^{4}$ Women with breast cancer may suffer from treatmentrelated side effects, such as surgical trauma, scarring, mastectomy and lymphedema. According to the medical data, ${ }^{56}$ these effects will easily lead to body image distortion, sexual dysfunction/intimacy problems as well as low self-esteem. Compared with the general population, when these patients are diagnosed with breast cancer, these effects will cause nearly $50 \%$ of patients with breast cancer to experience more considerable psychological distress, such as depression and anxiety, the prevalence of depression and anxiety in the 
year after diagnosis is around twice as high as in the general female population. ${ }^{78}$ Besides, lack of intimate confiding support will also lead to chronic depression and anxiety. The quality of life of patients with psychological symptoms is poor and the risk should not be ignored ${ }^{9-11}$ the depressive symptoms in patients with breast cancer can lead to physical deterioration and increased mortality. ${ }^{12} 13$ Early screening for depression is essential because of its severity.

However, complete screening for depression is difficult due to its complex aetiology and pathogenesis. Some previous studies have linked the prevalence of depression to the following factors, such as physical symptom burden, marital status, age, level of education, financial status and the number of therapies. ${ }^{14}{ }^{15}$ However, some of the results are inconsistent. Some researchers have shown that the chemotherapy can reduce the risk of depression, ${ }^{14}$ while others have found that the risk of depression is not affected by clinical factors such as prognosis, type of surgery or adjuvant radiotherapy, they consider that adjuvant chemotherapy may increase the risk of depression, anxiety or both during. ${ }^{16-20}$ Only after screening out unified risk factors, and looking for high-risk patients can we effectively provide more targeted treatment strategies and improve quality of life in patients with breast cancer and survivors. To provide strong evidence on the risk factors related to depression, we will conduct a systematic review of evidence-based medicine and a meta-analysis. The validated findings will give recommendations for physicians to identify patients with breast cancer with depression and the management of emotional problems for patients, and we can also give some suggestions on improving the quality of life of these patients.

\section{METHODS}

This study consists the Preferred Reporting Items for Systematic Reviews and Meta-Analyses (PRISMA) statement.

\section{Inclusion criteria for study selection}

Types of studies

Observational studies with available data on the prevalence and risk factors associated with anxiety and depression among patients with breast cancer will be considered. For study selection, we will exclude cross-sectional, cohort studies, case-control studies, case reports, case series, opinion papers, qualitative research, letters to the editor, comments, conference proceedings, policy documents, reviews and meta-analyses, study protocols without baseline data and animal studies.

\section{Types of patients}

Study population inclusion criteria will be all patients diagnosed with breast cancer, regardless of demographic age, race and education status.

Types of outcome measures

(1) Prevalence of anxiety and depression among patients with breast cancer; (2) Risk factors associated with anxiety and depression in patients with breast cancer and (3) The strength of the correlation between each risk factor and anxiety and depression.

\section{Search methods for the identification of studies \\ Data sources}

The following databases will be used: Web of Science, PubMed, EMBASE, Wan Fang Data Knowledge Service Platform, Chinese Biomedical Literature Database, Chinese Scientific Journal Database (VIP database), China National Knowledge Infrastructure. We will also conduct unpublished academic research data, contacting authors in the field for information. Two systematic reviews will be carried out from inception to February 2020. The reference lists of review articles will be conducted and the following search terms will be used: breast cancer, breast carcinoma, breast tumour, mammary cancer, mammary adenocarcinoma, anxiety, depression and depressive disorder. And we will use the search strategy provided in table 1 for searching the database. The authors will also search relevant trials from Clinical Trials.gov, Google Scholar and WHO International Clinical Trials Registry Platform.

\section{Study selection}

Studies imported into Endnote X9 software after deleting duplicates will be independently reviewed by two authors (JL and FZ) based on the exclusion and inclusion criteria. The researcher (WW) will read the full text of relevant articles to confirm the final inclusion of studies. For unclear study, the researcher (RP) will contact the author for details to determine whether this literature would be included. Any disagreement between reviewers will be resolved by discussion or a researcher (LJC). The study screening process is shown in figure 1. The documents selection will be demonstrated on a PRISMA flow chart.

\section{Risk of bias assessment}

The risk of bias will be assessed applying the Cochrane's 'Risk of bias' tool. The quality of the included studies will be assessed independently by two authors (QM and AZ) at the study and outcome levels. Any disagreements will be settled by discussion or with the arbitrament of the third author (LJ). In this study, we will use the NewcastleOttawa Scale to evaluate the quality of studies. This scale is a quality assessment tool for non-randomised controlled trials, with scores ranging from 0 to 9 ; scores of $0-4$ and 5-9 mean low quality and high quality, respectively.

\section{Statistical collection and analysis \\ Data extraction and management}

Extracted information include the first author's name, date of publication, journal, country and region, sample size ( $\mathrm{N}$ and male/female), duration of the study, baseline age, diagnostic criteria for breast cancer and anxiety and depression, incidence of anxiety and depression/ mean and SD for anxiety and depression score, variables, OR values, 95\% CI, and other relevant data for quality evaluation and risk of bias assessment. And the reasons 


\begin{tabular}{|c|c|}
\hline No & Search items \\
\hline 1 & observational study. Mesh. \\
\hline 2 & observational. ti.ab. \\
\hline 3 & observe. ti.ab. \\
\hline 4 & study. ti.ab. \\
\hline 5 & 1 or $2-4$ \\
\hline 6 & breast cancer. Mesh. \\
\hline 7 & breast carcinoma. ti.ab. \\
\hline 8 & breast tumor. ti.ab. \\
\hline 9 & mammary cancer. ti.ab. \\
\hline 10 & mammary adenocarinoma. ti.ab. \\
\hline 11 & 6 or $7-10$ \\
\hline 12 & anxiety. Mesh. \\
\hline 13 & anxious. ti.ab. \\
\hline 14 & hypervigilance. ti.ab. \\
\hline 15 & nervousness. ti.ab. \\
\hline 16 & social anxiety. ti.ab. \\
\hline 17 & anxieties, social. ti.ab. \\
\hline 18 & anxiety, social. ti.ab. \\
\hline 19 & social anxieties. ti.ab. \\
\hline 20 & 12 or $13-19$ \\
\hline 21 & depression. Mesh. \\
\hline 22 & depressions. ti.ab. \\
\hline 23 & depressive symptoms. ti.ab. \\
\hline 24 & depressive symptom. ti.ab. \\
\hline 25 & symptom, depressive. ti.ab. \\
\hline 26 & symptoms, depressive. ti.ab. \\
\hline 27 & emotional depression. ti.ab. \\
\hline 28 & depression, emotional. ti.ab. \\
\hline 29 & depressions, emotional. ti.ab. \\
\hline 30 & emotional depressions. ti.ab. \\
\hline 31 & 21 or $22-30$ \\
\hline 32 & 5 and 11 and 20 and 31 \\
\hline
\end{tabular}

for the exclusion of studies while extracting will also be recorded. The extraction of possible risk factors will be included gender, age, occupation, marital status, education level, social support, alcohol status, smoking status, pathological type, cancer clinical stage, disease course and therapy method. The extracted variables will be adjusted during the process, as it is likely that more and more variables that need to be included will turn up. Data collection will be done by two reviewers (JL and FZ) independently using Review Manager software. And if they are inconsistent in the process, they will discuss the results. A third reviewer will be consulted to resolve the doubts. For unclear details, the researchers will contact the corresponding authors by email for detailed information.

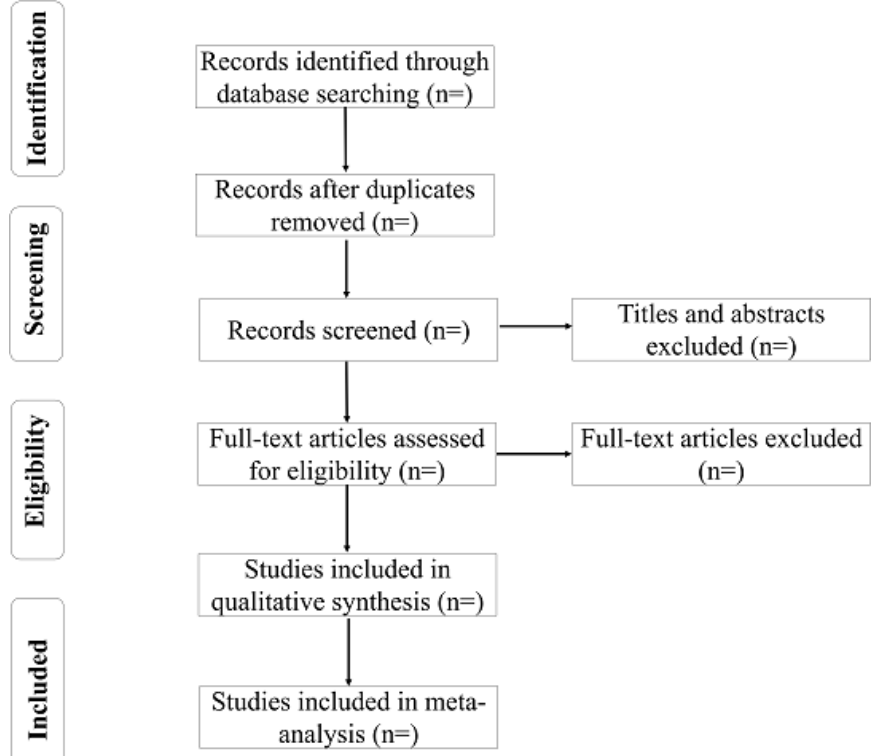

Figure 1 Flow diagram of the trial selection process.

\section{Measurements of prevalence and risk factors}

RevMan V.5.3 will be used to calculate the OR values and $95 \%$ CIs of the reported risk factors for anxiety and depression in breast cancer. When the CI for the OR does not include 1 , it is considered statistically significant. The prevalence estimates reported by the individual studies will be extracted or converted into prevalence percentages, and their respective SEs will be calculated. For the anxiety and depression scores, standardised mean difference will be used for analysis. We will use the FreemanTukey double arcsine transformation to stabilise the variance of study-specific prevalence. The prevalence of each study will be recalculated to confirm numerators and denominators, and adjustments as necessary.

Assessment of heterogeneity

The I $\mathrm{I}^{2}$ test will be used to determine the extent of heterogeneity. When the $\mathrm{I}^{2}$ value is less than $50 \%$, the fixedeffects model will be used. If the $\mathrm{I}^{2}$ value is higher than $50 \%$, the random-effects model will be used. In this study, factors of high heterogeneity will be removed one by one to identify the source of any observed heterogeneity. The causes of heterogeneity may include differences in study design, statistical methods and participants.

\section{Data synthesis}

We will use RevMan V.5.3 software for analysis. Metaanalysis will be performed when the heterogeneity is low or the source could be found, although heterogeneity is high. A systematic narrative synthesis will be conducted if it is impossible to complete any meta-analysis. If there is significant heterogeneity, we will use the subgroup analysis.

\section{Subgroup analysis}

Subgroup analysis will be done when data are available. The groups may be designed based on country or region, 
diagnostic criteria for anxiety and depression, bias score, time since diagnosis (long-term vs short-term survivors), severity/staging of breast cancer and study design.

\section{Meta-regression analysis}

Meta-regression analysis will be used to evaluate important factors (gender, age, occupation, marital status, education level, social support, alcohol status, smoking status, pathological type, cancer clinical stage, disease course and therapy method) on our study, which may explain heterogeneity across studies in the pooled effect size.

\section{Assessment of reporting biases}

Funnel plots will be used to assess publication bias. We will evaluate publication bias by performing the Begg's and Egger's tests. The significant $p$ value $(<0.05)$ indicates the existence of publication bias.

\section{Quality control of the systematic review and meta-analysis}

The methodological quality of the systematic review will be evaluated using the Measurement Tool to Assess Systematic Reviews. The Grading of Recommendations Assessment, Development and Evaluation will also be used to evaluate the strength of evidence produced by the systematic review (JL and FZ).

\section{DISCUSSION}

This study will review current researches to provide effective evidence on the risk factors related to depression and anxiety, and the results of systematic review and metaanalysis will provide significant help in identifying highrisk groups. These unified risk factors of depression will be screen out to advise on the management of emotional issues for patients. There may be some limitations to this review. First, a limitation will be the high heterogeneity studies may not be appropriate to be used in meta-analysis. Second, there may be heterogeneity in the diagnostic criteria for different types of anxiety and depression, as well as in the staging of cancer and depression. Different measurements and tools might lead to inconsistent levels of outcomes.

Contributors JL and FZ will identify eligible studies after reading titles and abstracts. WW will read the full texts to perform further selection. Several studies from different opinions will be determined by the RP. Data will be extracted from the original reports by LJC. The assessment of the risk of bias will be carried out by QM, AZ and JL. Any discrepancies will be resolved by discussion with a third AZ. JL and FZ will use the Grading of Recommendations Assessment, Development and Evaluation (GRADE) approach. JL conceived the review protocol and drafted the manuscript. FZ will monitor each procedure of the review. All authors read and approved the publication of the protocol.

Funding This work is supported by the National Natural Science Foundation of China (81973927).

Competing interests None declared.

Patient and public involvement Patients and/or the public were not involved in the design, or conduct, or reporting, or dissemination plans of this research.
Patient consent for publication Not required.

Provenance and peer review Not commissioned; externally peer reviewed.

Open access This is an open access article distributed in accordance with the Creative Commons Attribution Non Commercial (CC BY-NC 4.0) license, which permits others to distribute, remix, adapt, build upon this work non-commercially, and license their derivative works on different terms, provided the original work is properly cited, appropriate credit is given, any changes made indicated, and the use is non-commercial. See: http://creativecommons.org/licenses/by-nc/4.0/.

\section{ORCID iDs}

Jie Li http://orcid.org/0000-0002-6375-5123

Feng Zhang http://orcid.org/0000-0002-5557-0129

\section{REFERENCES}

1 Siegel RL, Miller KD, Jemal A. Cancer statistics, 2020. CA Cancer J Clin 2020;70:7-30.

2 Bray F, Ferlay J, Soerjomataram I, et al. Global cancer statistics 2018: GLOBOCAN estimates of incidence and mortality worldwide for 36 cancers in 185 countries. CA Cancer J Clin 2018;68:394-424.

3 Azamjah N, Soltan-Zadeh Y, Zayeri F. Global trend of breast cancer mortality rate: a 25-year study. Asian Pac J Cancer Prev 2019;20:2015-20.

4 Pinquart M, Fröhlich C, Silbereisen RK. Cancer patients' perceptions of positive and negative illness-related changes. J Health Psychol 2007;12:907-21.

5 Institute of Medicine US Cancer,, Policy Board. Meeting psychosocial needs of women with breast cancer. Washington, DC: National Academies Press (US), 2004.

6 Prates ACL, Freitas-Junior R, Prates MFO, et al. Influence of body image in women undergoing treatment for breast cancer. Rev Bras Ginecol Obstet 2017;39:175-83.

7 Okamura H, Watanabe T, Narabayashi M, et al. Psychological distress following first recurrence of disease in patients with breast cancer: prevalence and risk factors. Breast Cancer Res Treat 2000;61:131-7.

8 Burgess C, Cornelius V, Love S, et al. Depression and anxiety in women with early breast cancer: five year observational cohort study. BMJ 2005;330:702.

9 Kang D-H, Park N-J, McArdle T. Cancer-Specific stress and mood disturbance: implications for symptom perception, quality of life, and immune response in women shortly after diagnosis of breast cancer. ISRN Nurs 2012;2012:1-7.

10 Fann JR, Thomas-Rich AM, Katon WJ, et al. Major depression after breast cancer: a review of epidemiology and treatment. Gen Hosp Psychiatry 2008;30:112-26.

11 Perry S, Kowalski TL, Chang C-H. Quality of life assessment in women with breast cancer: benefits, acceptability and utilization. Health Qual Life Outcomes 2007;5:24.

12 Liang X, Margolis KL, Hendryx M, et al. Effect of depression before breast cancer diagnosis on mortality among postmenopausal women. Cancer 2017;123:3107-15.

13 Jacob L, Kalder M, Kostev K. Incidence of depression and anxiety among women newly diagnosed with breast or genital organ cancer in Germany. Psychooncology 2017;26:1535-40.

14 Cvetković J, Nenadović M. Depression in breast cancer patients. Psychiatry Res 2016;240:343-7.

15 McFarland DC, Shaffer KM, Tiersten A, et al. Physical symptom burden and its association with distress, anxiety, and depression in breast cancer. Psychosomatics 2018;59:464-71.

16 Burgess CC, Ramirez AJ, Richards MA, et al. Does the method of detection of breast cancer affect subsequent psychiatric morbidity? Eur J Cancer 2002;38:1622-5.

17 Kiebert GM, de Haes JC, van de Velde CJ. The impact of breastconserving treatment and mastectomy on the quality of life of earlystage breast cancer patients: a review. J Clin Oncol 1991;9:1059-70.

18 Lee MS, Love SB, Mitchell JB, et al. Mastectomy or conservation for early breast cancer: psychological morbidity. Eur J Cancer 1992;28A:1340-4.

19 Hughson AV, Cooper AF, McArdle CS, et al. Psychological impact of adjuvant chemotherapy in the first two years after mastectomy. $\mathrm{Br}$ Med J 1986;293:1268-71.

20 Dean C. Psychiatric morbidity following mastectomy: preoperative predictors and types of illness. J Psychosom Res 1987;31:385-92. 